\title{
Altered levels of cytokines in patients with irritable bowel syndrome are not correlated with fatigue
}

This article was published in the following Dove Press journal: International Journal of General Medicine

\author{
Ellen Johanne Vara' \\ Karl A Brokstad ${ }^{2}$ \\ Trygve Hausken 1,3,4 \\ Gülen Arslan Lied 1,3,4 \\ 'Centre of Nutrition, Department \\ of Clinical Medicine, University \\ of Bergen, Bergen, Norway; \\ ${ }^{2}$ Broegelmann Research Laboratory, \\ Department of Clinical Science, \\ University of Bergen, Bergen, Norway; \\ ${ }^{3}$ Section of Gastroenterology, \\ Haukeland University Hospital, \\ Bergen, Norway; ${ }^{4}$ National Centre of \\ Functional Gastrointestinal Disorders, \\ Haukeland University Hospital, \\ Bergen, Norway
}

Introduction: A considerable number of patients with gastrointestinal complaints attributable to irritable bowel syndrome (IBS) have shown evidence of immune activation. Fatigue is also frequently reported by IBS patients and the condition is considered as a common comorbidity of IBS. Therefore, it is interesting to see whether these two conditions share the same pathophysiological mechanism. Aims: To investigate the potential role of cytokine profiles in patients with IBS and the relationship between cytokine profiles and fatigue.

Materials and methods: Thirty-eight patients with IBS ( 32 females, 6 males, age range 18-70 years) and 22 healthy individuals (control group) (17 females, 5 males, age range 24-42 years) were included. IBS was diagnosed according to Rome III criteria, and severity of IBS symptoms and fatigue were evaluated using the Irritable Bowel Syndrome-Severity Scoring System (IBSSSS) and Fatigue Impact Scale (FIS), respectively. FIS scores of 25 or higher were defined as fatigue. Blood samples were also taken, and the Luminex ${ }^{\circledR}$ platform (Cytokine Human Ultrasensitive Magnetic 10-Plex Panel) was used for quantifying human cytokines' profile (granulocytemacrophage colony-stimulating factor, interferon- $\gamma$, interleukin [IL]-1 $\beta$, IL-2, IL-4, IL-5, IL-6, IL-8, IL-10, and tumor necrosis factor [TNF]- $\alpha$ ) in serum.

Results: The serum levels of IL-5, IL-6, IL-10, and TNF- $\alpha$ were significantly higher in patients with IBS compared to healthy controls ( $p=0.003, p=0.011, p=0.007$, and $p=0.02$, respectively). Conversely, serum levels of cytokine IL- $1 \beta$ were significantly higher in the control group $(p=0.03)$. The findings were consistent when comparing nonatopic patients with controls. Fatigue was demonstrated in $84.2 \%$ of the IBS patients. Scores of IBS-SSS were not significantly correlated with FIS scores $(r=0.2, p=0.19)$, and they were not significantly different in patients with FIS scores $>25$ compared to patients with FIS scores $<25(p=0.11)$. None of the cytokine levels were significantly different in IBS patients with FIS scores $>25$ compared to IBS patients with FIS scores $<25$. Moreover, the cytokine levels in participants did not vary significantly between patients with diarrhea, constipation, or mixed bowel habits in multiple comparisons of patients. Conclusions: The cytokines IL-5, IL-6, IL-10, and TNF- $\alpha$ may contribute to the development of IBS. However, serum levels of cytokines were not significantly different in IBS patients with fatigue compared with IBS patients without fatigue. Thus, the significance of cytokine levels may be less important than anticipated in search of common underlying mechanisms, and other factors should be explored in future studies.

Keywords: irritable bowel syndrome, fatigue, proinflammatory cytokines, immune activation

\section{Introduction}

Irritable bowel syndrome (IBS) is a common functional gastrointestinal (GI) disorder with a prevalence rate of $\sim 10 \%-15 \%$ in the Western world. ${ }^{1,2}$ The condition is characterized
Correspondence: Gülen Arslan Lied Centre of Nutrition, Department of Clinical Medicine, University of Bergen, Postboks 7804, 5020, Bergen, Norway Tel +4755973058

Email Gulen.Arslan@uib.no 
by pain or discomfort in the abdomen, flatulence, and altered bowel habits as diarrhea, constipation, or changing between constipation and diarrhea. In addition to abdominal symptoms, IBS is commonly associated with extraintestinal symptoms and comorbidities, such as fatigue and fibromyalgia., ${ }^{1,34}$ Fatigue symptoms are described by patients as a long-lasting tiredness or weakness and can be experienced both physically and mentally. Fatigue is often observed to have a vast impact on patients' daily life and is associated with decreased quality of life. ${ }^{5}$ In addition, fatigue is experienced by many patients with IBS, and patients with functional GI disorders often report more severe fatigue than patients with organic disease and controls. ${ }^{1,5,6}$ The underlying mechanisms of fatigue are proven to be both biological and psychological, and the proposed factors are immunological, such as proinflammatory cytokines, and genetically susceptible. ${ }^{7}$ Studies in patients with chronic fatigue have shown improvement of symptoms after B-cell depletion therapy, supporting the hypothesis of a potential immune dysfunction in patients with fatigue. ${ }^{8}$

A considerable number of patients with GI complaints compatible with IBS symptoms have shown evidence of immune activation. ${ }^{2,9}$ Measurements of the immune system have also been studied in search for fatigue biomarkers, and evidence from a previous study has shown that inflammation and immune activation play an important role in the development of IBS. ${ }^{8}$ Moreover, studies from Haukeland University Hospital have reported an increased development of IBS symptoms and chronic fatigue in patients after Giardia lamblia infection. ${ }^{10-13}$ The study concluded that patients infected with the bacteria developed postinfectious IBS, suggesting that activation of the immune system plays an important role in the development of GI symptoms and fatigue in IBS patients. Consequently, the question is how the immune system is activated in IBS patients and if this affects symptoms of fatigue. A reduced expression of genes linked to cytokine secretion for a number of key cytokines has been observed in the colonic mucosa of patients reporting IBS symptoms, indicating that immunological differences in the human intestinal mucosa could induce GI complaints. ${ }^{14}$

As a result of observing familiar immunological factors in patients with fatigue and IBS, a common underlying pathophysiological mechanism explaining these comorbidities has been proposed. Thus, the aims of this study were to investigate the potential role of cytokines, granulocyte-macrophage colony-stimulating factor (GM-CSF), interferon (IFN)- $\gamma$, interleukin (IL)-1 $\beta$, IL-2, IL-4, IL-5, IL-6, IL-8, IL-10, and TNF- $\alpha$, in patients with IBS and to examine if cytokine levels were different in IBS patients with fatigue compared with IBS patients without fatigue.

\section{Materials and methods}

\section{Subjects}

Thirty-eight patients referred to Section of Gastroenterology, Department of Medicine at Haukeland University Hospital for GI complaints were asked to participate. Patients filled in validated questionnaires to assess GI symptoms and fatigue during their consultations. Patients with recent intake of nonsteroidal anti-inflammatory drugs, organic diseases, and pregnant and lactating women were excluded. No age criteria were addressed.

In addition, a control group consisted of 22 volunteers who claimed to be healthy was included. They filled in questionnaires regarding abdominal complaints (Irritable Bowel Syndrome-Severity Scoring System [IBS-SSS]) and allergic diseases including a history of atopy, but they were not examined otherwise.

All patients and controls received written and oral information, and participating patients provided a signed consent. The study has been approved by the Regional Committees for Medical and Health Research Ethics (REC West, Norway).

\section{Questionnaires}

The following questionnaires were filled out by participating patients: 1) the short form of the Rome III criteria for diagnosing of IBS, ${ }^{15}$ 2) the IBS-SSS to assess severity of IBS symptoms, ${ }^{16}$ and 3 ) the Fatigue Impact Scale (FIS) to grade the severity of chronic fatigue.

The IBS-SSS contains five questions regarding frequency and intensity of abdominal pain, severity of abdominal distension, dissatisfaction with bowel habits, and interference of IBS with daily life. The questions were rated on 100-point visual analog scales, with a maximum score of $500 .{ }^{16}$ All five components contributed equally. It has been established in previous studies that IBS-SSS scores $<75$ are equivalent to no IBS, scores $<175$ indicate mild IBS symptoms, scores ranging from 175 to 300 indicate moderate symptoms, and scores $>300$ represent severe IBS. ${ }^{16}$ Patients were also subclassified into diarrhea-predominant (IBS-D) group, constipation-predominant (IBS-C) group, and those with mixed/alternating bowel habits (IBS-M).

The FIS questionnaire is considered to be a reliable and valid tool to measure the influence of fatigue in patients. It is a two-part questionnaire used to assess the impact of fatigue on the patient's daily life. ${ }^{17}$ The scale is constructed to include three subscales assessing perceived fatigue impact on cognitive functioning (10 items), physical functioning (10 items), and psychosocial functioning (20 items). The statements are ranged on a five-level scale $(0=$ no problem 
to 4 = extreme problems), giving a maximum FIS score of 160. A FIS score $\geq 25$ was defined as fatigue in accordance with a previous study. ${ }^{6}$

\section{Blood samples}

In all participants, blood samples were taken for measuring levels of cytokines and allergen-specific immunoglobulin $\mathrm{E}$ (IgE) antibodies in serum. Gel tubes were used to take blood samples for cytokine analysis in serum. The samples were centrifuged 30 minutes within 2 hours after phlebotomy, and serum for later analyses was frozen at $-80^{\circ} \mathrm{C}$ degrees. The Cytokine Human Ultrasensitive Magnetic 10-Plex Panel for the Luminex ${ }^{\circledR}$ platform (Thermo Fisher Scientific, Oslo, Norway) was used for quantification of cytokines GM-CSF, IFN- $\gamma$, IL-1 $\beta$, IL-2, IL-4, IL-5, IL-6, IL-8, IL-10, and TNF- $\alpha$ in serum. They were measured according to the manufacturer's instructions and expressed as $\mathrm{pg} / \mathrm{mL}$.

Specific IgE antibodies to inhalant allergens were also measured using Phadiatop ${ }^{\odot}$ (birch, timothy, Cladosporium herbarum, cat, dog, horse, mugwort, and Dermatophagoides pteronyssinus) in the patient group, and atopic sensitization was defined as having increased levels of $\mathrm{IgE}$ antibodies $(\geq 0.35 \mathrm{kUA} / \mathrm{L})$ to at least one allergen.

\section{Statistical analyses}

All statistical analyses were performed using the GraphPad Prism 6 statistical software package (GraphPad Software, Inc., San Diego, CA, USA) and Statistical Product and Service Solutions, version 25 (IBM SPSS Statistics 21 Inc., Chicago, IL, USA). Nonparametric statistical tests were conducted when analyzing the data material. Descriptive statistics were calculated and pairwise comparisons between groups were performed with the Mann-Whitney $U$-test. The continuous variables from the Mann-Whitney $U$-test are reported as medians (25th-75th percentiles). Correlations between different parameters within the same group were evaluated by Spearman's correlation coefficient. Linear regression models were used to evaluate the association between scores of IBS-SSS and FIS, adjusting for gender and age in multivariate regression models. Kruskal-Wallis tests were performed for multiple comparisons between variables. All tests were two-tailed and the level of significance was set to $p<0.05$.

\section{Results \\ Sample characteristics}

Clinical and demographic data for the patient group are presented in Table 1.
Table I Demographic and clinical data in the patient cohort

\begin{tabular}{ll}
\hline Characteristics & \\
\hline Patients, total number & 38 \\
Females & $84.2 \%(n=32)$ \\
Males & $15.8 \%(n=6)$ \\
Age (years), mean (range) & $34.7(18-70)$ \\
Duration of symptoms (years), mean (range) & $8.2(I-30)$ \\
Atopic sensitized & $50 \%(n=19)$ \\
IBS-SSS, mean (range) & $288.8(70-440)$ \\
$\geq 300$ (severe) & $39.5 \%(n=15)$ \\
I75-300 (moderate) & $52.6 \%(n=20)$ \\
$75-<175$ (mild) & $5.3 \%(n=2)$ \\
$<75$ (no IBS) & $2.6 \%(n=1)$ \\
IBS subgroups & \\
Predominant diarrhea & $34.2 \%(n=13)$ \\
Predominant constipation & $21.1 \%(n=8)$ \\
Mixed constipation/diarrhea & $44.7 \%(n=17)$ \\
Fatigue & \\
Mean FIS (range) & $69.8(9-157)$ \\
FIS $\geq 25$ & $84.2 \%(n=32)$ \\
\hline
\end{tabular}

Abbreviations: FIS, Fatigue Impact Scale; IBS, irritable bowel syndrome; IBS-SSS, IBS-severity scoring system.

A control group of 22 healthy individuals were included. The mean age among healthy individuals was 32 years (age range: $24-42$ years) and $77 \%$ of them were women (17 female and 5 men).

\section{Profile of cytokines}

The serum levels of IL-5, IL-6, IL-10, and TNF- $\alpha$ were significantly higher in patients with IBS compared with a healthy control group ( $p=0.003, p=0.011, p=0.007$, and $p=0.02$, respectively; Figure 1). Conversely, serum levels of cytokine IL-1 $\beta$ were significantly higher in the control group $(p=0.03)$. Figure 2 illustrates the spread of IL- $1 \beta$ in the patient group and among controls. Otherwise, other cytokines, such as GM-CSF, IFN- $\gamma$, IL-2, IL-4, and IL-8, did not significantly differ between the groups.

No significant correlations were observed between IBSSSS scores and levels of cytokines in serum in IBS patients. However, correlation analysis revealed significant correlations between age and serum levels of IL- 4 and IL-5 in the patient group ( $p=0.04$ and 0.004 , respectively).

Serum levels of cytokine did not differ significantly when comparing female patients with male patients, nor when comparing patients with diarrhea to patients with constipation and mixed bowel habits in multiple comparisons.

\section{Fatigue and IBS-symptom severity scores}

Thirty-two patients (84.2\%) had fatigue as having FIS score $\geq 25$. No significant correlation was observed between scores of IBS-SSS and FIS in the patient group $(r=0.2, p=0.19$; 

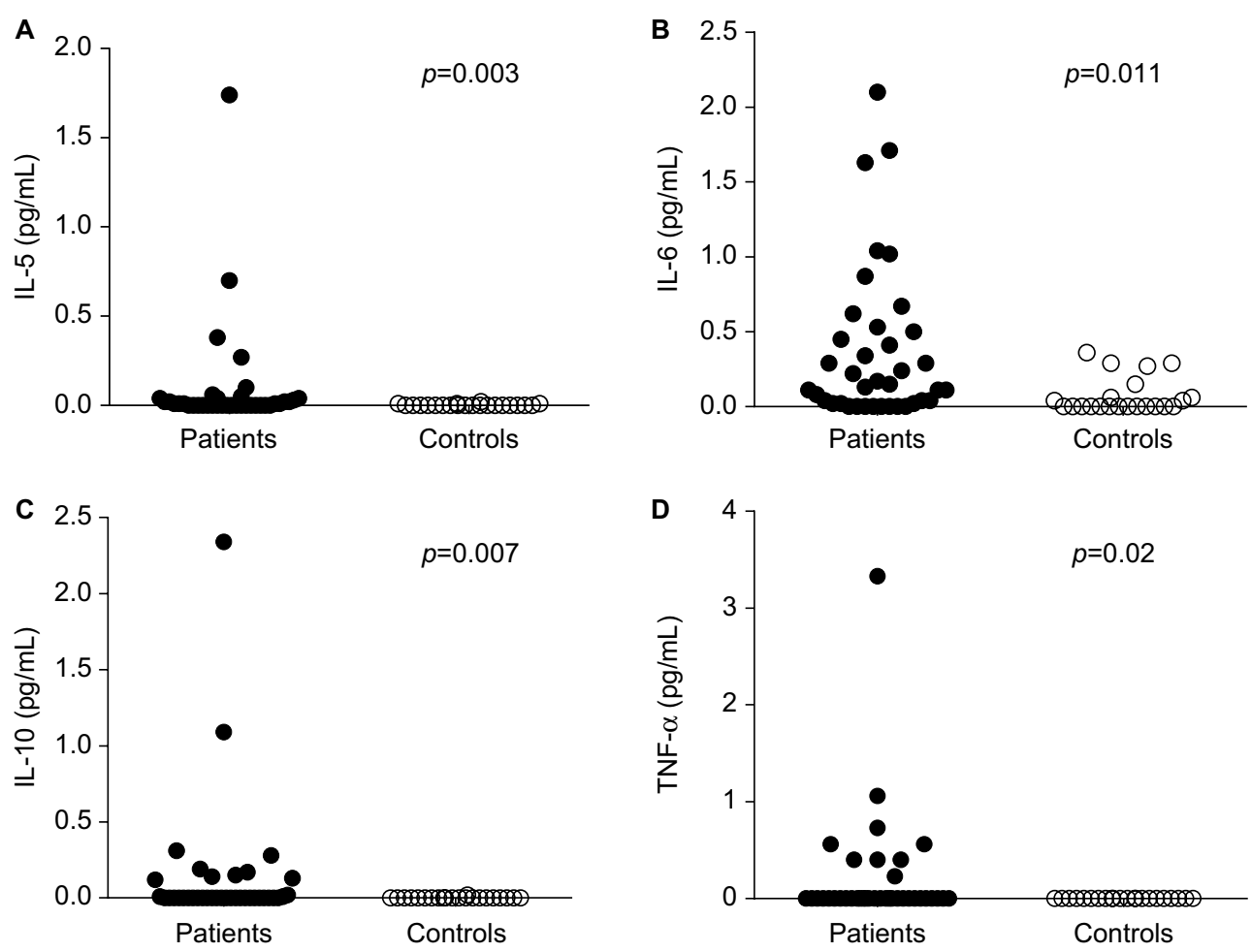

Figure I The serum levels of cytokines IL-5 (A), IL-6 (B), IL-I0 (C), and TNF- $\alpha$ (D) in patients with irritable bowel syndrome and controls. Abbreviations: IL, interleukin; TNF, tumor necrosis factor.

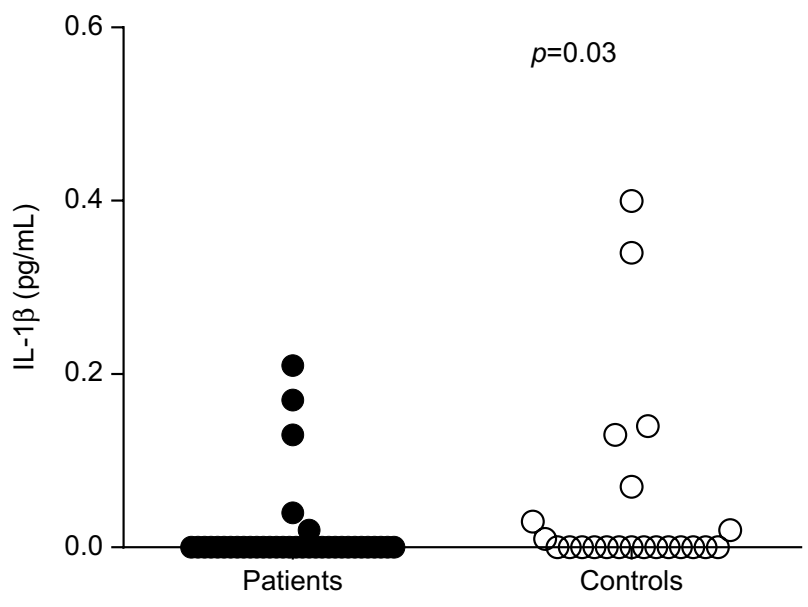

Figure 2 The serum levels of cytokine IL-I $\beta$ in patients with irritable bowel syndrome and controls.

Abbreviation: IL, interleukin.

Figure 3). FIS scores were not significantly different in patients with FIS scores $\geq 25$ compared to patients with FIS scores $<25$. Moreover, FIS scores were not significantly associated with IBS-SSS scores in the patient group in a multivariate regression analysis adjusting for sex and age ( $b=0.373, p=0.28$ ).

\section{Fatigue and cytokines}

All cytokines levels have been compared according to degree of fatigue, and none of the serum levels of cytokines were significantly different in patients with fatigue (FIS scores $\geq 25$ ) compared with patients without fatigue (FIS scores $<25$ ).

\section{Analysis of cytokine levels in stratified patient group}

Stratified patient sampling according to atopic sensitization was performed in order to investigate significant associations in nonatopic patients. Consistent with previous findings, serum levels of cytokines IL-5, IL-6, IL-10, and TNF- $\alpha$ were higher in patients, whereas IL- 6 and TNF- $\alpha$ were found to be significantly higher in patients compared with controls ( $p=0.008$ and $p=0.015$, respectively). In addition, serum levels of cytokine IL- $1 \beta$ were significantly higher in the control group compared with nonatopic IBS patients $(p=0.02)$.

\section{Discussion}

This study attempted to find a common pathophysiological mechanism by investigating the potential role of cytokine profiles in the development of IBS and the relationship with 


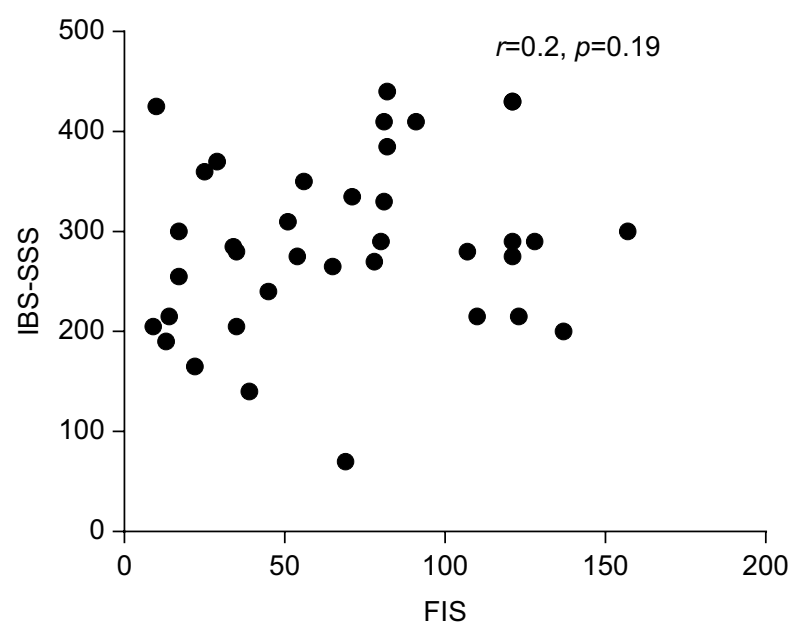

Figure 3 The correlation between IBS-SSS and FIS scores in patients with irritable bowel syndrome (nonsignificant; $r=0.2, p=0.19$ ).

Abbreviations: FIS, Fatigue Impact Scale; IBS-SSS, Irritable Bowel SyndromeSeverity Scoring System.

fatigue, which is one of the comorbidities of IBS. Among the 10 different proinflammatory cytokines, the serum levels of IL-5, IL-6, IL-10, and TNF- $\alpha$ were significantly higher in patients with IBS, but the serum levels of IL-1 $\beta$ were significantly higher in healthy individuals when they were compared. The findings were consistent when comparing nonatopic patients with controls. None of the cytokine levels were significantly different in IBS patients with and without fatigue. Moreover, the cytokine levels did not vary significantly between genders and between patients with diarrhea, constipation, and mixed bowel habits in multiple comparisons of patients.

Fatigue is one of the most common "extraintestinal" complaints of patients with IBS, but it is still poorly understood. The biggest causes of health care costs of patients with IBS are non-GI symptoms (eg, fatigue, depression, and anxiety). Fatigue in IBS was described with a wide range of definitions, such as tiredness, extreme weariness, and a state of exhaustion. Fatigue is likely to interfere with work productivity, healthrelated quality of life, cost effectiveness, and daily activity in this patient group. ${ }^{8}$ It is challenging to provide effective fatigue management for patients with IBS because there is a lack of research and information about fatigue compared with other IBS symptoms, such as abdominal pain, diarrhea, constipation, and psychological distress. ${ }^{8}$ Furthermore, IBS and the most common comorbidities share a number of demographic and clinical features, such as a predominance of female gender, the presence of enhanced fatigue, and sleep disturbances, and the occurrence of psychological comorbidities, such as anxiety and depression. ${ }^{6}$ This could lead one to speculate that all of these disorders share a common underlying pathophysiological mechanism. However, the underlying mechanisms still remain unclear, although previous studies have revealed immune activation in IBS patients, suggesting that immunological alterations or imbalances could constitute causes of inducing symptoms of IBS as well as fatigue., , $14,18,19^{2}$ Recently, we examined the association between functional GI complaints and levels of total IgE in serum of IBS patients with extraintestinal symptoms; however, no significant associations were found. ${ }^{20}$ Thus, in compliance with previous studies implying associations between proinflammatory cytokines and IBS, ${ }^{19}$ we wanted to explore the association between the immunological cytokines GM-CSF, IFN- $\gamma$, IL-1 $\beta$, IL-2, IL-4, IL-5, IL-6, IL-8, IL-10, and TNF- $\alpha$ in serum and the comorbidities of IBS and fatigue. We found that patients with IBS had significantly higher serum levels of IL-5, IL-6, IL-10, and TNF- $\alpha$, and significantly lower serum levels of IL-1 $\beta$ compared with controls. This is not in line with previous studies as they found low serum levels of IL-10 and high serum levels of IL-1 $\beta$ in IBS patients. ${ }^{21,22}$ Nevertheless, all these suggest that there is a kind of abnormal immune regulation, supporting the presence of immune activation in IBS patients. However, we do not yet know how the immune system is activated and it is, therefore, difficult to draw any conclusion from the findings. In addition, in our study, no significant associations were found between cytokine levels and IBS-SSS, or between cytokine levels and fatigue. This could indicate that there is no significant association between plasma cytokine profiles and symptom generation in IBS patients with fatigue. This is in accordance with previous studies ${ }^{19,23}$ and may suggest that cytokine levels have a less important role in determining the nature of the comorbidities of fatigue and IBS than predicted. Even though Scully et al found increased serum levels of IL- $1 \beta$ and TNF- $\alpha$ in IBS patients with extraintestinal comorbidities (fibromyalgia, premenstrual dysmorphic disorder, and chronic fatigue syndrome), ${ }^{19}$ there was no evidence for associations between cytokine profiles and the nature of the comorbidity or the number of extraintestinal comorbidities present.

Newer studies are linking GI complaints and immune dysfunctions with alterations of the gut microbiota. ${ }^{19}$ It is well known that the bacterial flora colonizing the GI tract play a major role in processing nutrients, regulating, and maintaining the immune system, as well as having a central nervous system function, for example, the "gut-brain" axis. Moreover, overgrowth or imbalance of specific bacterial strains have been associated with a variety of disorders, such as IBS, Crohn's disease, allergies, and other autoimmune 
diseases. ${ }^{24,25}$ Thus, it is conceivable that alterations in the gut microbiota have clinical significance in the pathophysiology and the symptom generation of IBS, and more research is, therefore, warranted in order to see alterations in gut microbiota in IBS patients with and without extraintestinal comorbidities.

Patients included in this study comprised a consistent group, with a high prevalence of moderate and severe IBS symptoms, and they had a high prevalence of fatigue (84.2\%) corresponding with our previous study. ${ }^{20}$ The small size of the patient sample and the range of the study populations are weaknesses of this study, whereas the control group is smaller than the patient group even though mean age values were similar in both groups. Future studies with larger patient and control groups are, therefore, of interest, in order to further reveal the association between cytokine profiles and the comorbidity of IBS and fatigue.

\section{Conclusion}

The cytokines IL-5, IL-6, IL-10, and TNF- $\alpha$ may contribute to the development of IBS. However, serum levels of cytokines were not significantly different in IBS patients with fatigue compared with IBS patients without fatigue. The cytokine profiles do not appear to be involved in the pathogenesis of individual extraintestinal comorbid conditions that may accompany IBS. Thus, the significance of cytokine levels may be less important than anticipated in search of common underlying mechanisms, and other factors including alterations in gut microbiota diversity and their metabolites should be explored in future studies.

\section{Disclosure}

The authors report no conflicts of interest in this work.

\section{References}

1. Frandemark Å, Jakobsson Ung E, Tornblom H, Simren M, Jakobsson S. Fatigue: a distressing symptom for patients with irritable bowel syndrome. Neurogastroenterol Motil. 2017;29(1):e12898.

2. Farup PG, Ueland T, Rudi K, Lydersen S, Hestad K. Functional bowel disorders are associated with a central immune activation. Gastroenterol Res Pract. 2017;2017:1642912.

3. Piche T, Huet PM, Gelsi E, et al. Fatigue in irritable bowel syndrome: characterization and putative role of leptin. Eur J Gastroenterol Hepatol. 2007;19(3):237-243.

4. Berstad A, Undseth R, Lind R, Valeur J. Functional bowel symptoms, fibromyalgia and fatigue: a food-induced triad? Scand J Gastroenterol. 2012;47(8-9):914-919.

5. Simrén M, Simrén J, Posserud I, Bjornsson ES, Abrahamsson H. Predictors of subjective fatigue in chronic gastrointestinal disease. Aliment Pharmacol Ther. 2008;28(5):638-647.
6. Lind R, Berstad A, Hatlebakk J, Valeur J. Chronic fatigue in patients with unexplained self-reported food hypersensitivity and irritable bowel syndrome: validation of a Norwegian translation of the Fatigue Impact Scale. Clin ExpGastroenterol. 2013;6:101-117.

7. Norheim KB, Jonsson G, Omdal R. Biological mechanisms of chronic fatigue. Rheumatology (Oxford). 2011;50(6):1009-1018.

8. Lunde S, Kristoffersen EK, Sapkota D, et al. Serum BAFF and APRIL Levels, T-lymphocyte subsets, and immunoglobulins after B-cell depletion using the monoclonal anti-CD20 antibody rituximab in myalgic encephalopathy/chronic fatigue syndrome. PLoS One. 2016;11(8):e0161226.

9. Lied GA, Lillestol K, Lind R, et al. Perceived food hypersensitivity: a review of 10 years of interdisciplinary research at a reference center. Scand J Gastroenterol. 2011;46(10):1169-1178.

10. Hanevik K, Dizdar V, Langeland N, Hausken T. Development of functional gastrointestinal disorders after Giardia lamblia infection. $B M C$ Gastroenterol. 2009;9:27.

11. Kumar S, Shukla R, Ranjan P, Kumar A. Interleukin-10: a compelling therapeutic target in patients with irritable bowel syndrome. Clin Ther. 2017;39(3):632-643.

12. Hanevik K, Kristoffersen E, Mørch K, et al. Giardia-specific cellular immune responses in post-giardiasis chronic fatigue syndrome. $B M C$ Immunol. 2017;18(1):5.

13. Wensaas KA, Langeland N, Hanevik K, Mørch K, Eide GE, Rortveit G. Irritable bowel syndrome and chronic fatigue 3 years after acute giardiasis: historic cohort study. Gut. 2012;61(2):214-219.

14. Macsharry J, O'Mahony L, Fanning A, et al. Mucosal cytokine imbalance in irritable bowel syndrome. Scand J Gastroenterol. 2008;43(12):1467-1476.

15. Longstreth GF, Thompson WG, Chey WD, Houghton LA, Mearin F, Spiller RC. Functional bowel disorders. Gastroenterology. 2006;130(5):1480-1491.

16. Francis CY, Morris J, Whorwell PJ. The irritable bowel severity scoring system: a simple method of monitoring irritable bowel syndrome and its progress. Aliment Pharmacol Ther. 1997;11(2):395-402.

17. Fisk JD, Ritvo PG, Ross L, Haase DA, Marrie TJ, Schlech WF. Measuring the functional impact of fatigue: initial validation of the fatigue impact scale. Clin Infect Dis. 1994;18(Suppl 1):S79-S83.

18. Bashashati M, Rezaei N, Shafieyoun A, et al. Cytokine imbalance in irritable bowel syndrome: a systematic review and meta-analysis. Neurogastroenterol Motil. 2014;26(7):1036-1048.

19. Scully P, McKernan DP, Keohane J, et al. Plasma cytokine profiles in females with irritable bowel syndrome and extra-intestinal co-morbidity. Am J Gastroenterol. 2010;105(10):2235-2243.

20. Vara EJ, Valeur J, Hausken T, Lied GA. Extra-intestinal symptoms in patients with irritable bowel syndrome: related to high total IgE levels and atopic sensitization? Scand J Gastroenterol. 2016;51(8):908-913.

21. Choghakhori R, Abbasnezhad A, Hasanvand A, Amani R. Inflammatory cytokines and oxidative stress biomarkers in irritable bowel syndrome: association with digestive symptoms and quality of life. Cytokine. 2017;93:34-43.

22. Schmulson M, Pulido-London D, Rodriguez O, et al. Lower serum IL-10 is an independent predictor of IBS among volunteers in Mexico. $\mathrm{Am} J$ Gastroenterol. 2012;107(5):747-753.

23. Bennet SM, Polster A, Tornblom H, et al. Global cytokine profiles and association with clinical characteristics in patients with irritable bowel syndrome. Am J Gastroenterol. 2016;111(8):1165-1176.

24. Fremont M, Coomans D, Massart S, De Meirleir K. High-throughput 16S rRNA gene sequencing reveals alterations of intestinal microbiota in myalgic encephalomyelitis/chronic fatigue syndrome patients. Anaerobe. 2013;22:50-56.

25. Bhattarai Y, Muniz Pedrogo DA, Kashyap PC. Irritable bowel syndrome: a gut microbiota-related disorder? Am J Physiol Gastrointest Liver Physiol. 2016;312(1):G52-G62. 
The International Journal of General Medicine is an international, peer-reviewed open-access journal that focuses on general and internal medicine, pathogenesis, epidemiology, diagnosis, monitoring and treatment protocols. The journal is characterized by the rapid reporting of reviews, original research and clinical studies across all disease areas.
The manuscript management system is completely online and includes a very quick and fair peer-review system, which is all easy to use. Visit http://www.dovepress.com/testimonials.php to read real quotes from published authors. 